Japan. J. Math.

Vol. 6. No. 1, 1980

\title{
Non-catenary pseudo-geometric normal rings
}

\author{
By Tetsushi Ogoma
}

(Received December 20, 1978)

\section{Introduction}

It is known for long that geometric rings are catenary (i.e. they satisfy the chain condition for prime ideals), but it had been a problem whether general noetherian rings are catenary.

In 1956, Nagata showed a counter example in [5] for the first time. This example is constructed as a subring of an unequidimensional catenary ring which is the normalization of the former. Therefore, in his investigation, catenarity and equidimensionality of integral extensions are important, which he calls the second chain condition, and he proved in [5] that formally equidimensional local rings satisfy the second chain condition.

From these results, the problem a rose whether noetherian normal local rings satisfy the second chain condition or not. Many authors studied this. For example, Ratliff showed in [8] the equivalence of formal equidimensionality, dimension formula and the second chain condition for local domains. Later, Seydi showed in [10] the equivalence of catenarity, universal catenarity and the second chain condition for henselian local domains. Nevertheless it remained unsolved whether normal domains or henselian rings are catenary or not.

We give in this note a negative answer for it by showing an example. Consequently in the pseudo-geometric cases, in particular, we can settle the problem as follows:

Reasons for rings not to be universally catenary are classified, which we call obstructions. Namely, if a pseudo-geometric ring $R$ is not universally catenary, then $R$ has an obstruction which is integrally solvable or formal. An obstruction of $R$ with locally equidimensional generic formal fibers is called integrally solvable, because normalization and henselization of $R$ are universally catenary. Otherwise, the obstruction is called formal and can not be solved by any (f.q.)-integral extensions.

In appendix, we shall show by an example that in order to discriminate obstructions by local formal fibers, it is important to assume that the rings under consideration are pseudo-geometric. This example $T$ is very inter- 
esting by itself. Indeed, $T / z T$ is a domain whose completion has an imbedded prime divisor. (Problem of Nagata in [5]; the existence was shown by Ferrand and Raynaud in [1].) Moreover, $T$ is Japanese but not universally Japanese. Adopting our construction of $T$, and interchanging $\boldsymbol{Q}$ by $\boldsymbol{F}_{p}$ if necessary, we can construct interesting examples which have curious formal fibers.

In this note, we understand by a ring, a commutative ring with identity. A noetherian ring $R$ is called pseudo-geometric (called "universally Japanese" in [2] or "Nagata" in [3]) if the integral closure of $R / p$ in an arbitrary finite algebraic extension of the field $k(\mathfrak{p})=R_{\mathfrak{p}} / \mathfrak{p} R_{\mathfrak{p}}$ is a finite $R / \mathfrak{p}$-module for every $\mathfrak{p} \in \operatorname{Spec} R$. It is well known ([4] (36.4)) that if $R$ is a pseudo-geometric local domain, then the completion $\hat{R}$ of $R$ is reduced (i.e. nilpotent free).

The terminologies and notations of [2], [3] and [4] will be used freely. In particular, for local rings, ^ ( $\hat{R}$ etc.) indicates completion. If $p \in \operatorname{Spec} R$, $k(\mathfrak{p})$ denotes the field of fractions of $R / \mathfrak{p}$.

The writer wishes to thank Prof. M. Nagata, Prof. T. Akiba and Prof. J. Nishimura for their critical reading of this note. He also wishes to thank Dr. C. Rotthaus for her method in [9] of constructing a pseudo-geometric local ring whose regular locus is not open, because her method is adopted to our example in $\S 3$.

\section{$\S$ I. Characterization in completion by generic formal fibers for pseudo-geometric normal local domains}

It is well known that if a noetherian normal local domain $R$ has normal formal fibers, then $\hat{R}$ is normal ([3] (21.E)). On the other hand, it is not known whether $\hat{R}$ has a property, say $(P)$, if formal fibers have $(P)$, where $(P)$ is a condition weaker than normal. We shall give affirmative answers if $R$ is a pseudo-geometric normal domain and if $(P)$ is "locally irreducible"" or "locally equidimensional".

We begin with a preliminary lemma ${ }^{2}$. By the depth of a finite $R$-module $M$ for local ring $(R, \mathfrak{m})$, we mean the length of a maximal $M$-regular sequence, which is characterized as the number $n$ such that $\operatorname{Ext}_{R}^{i}(R / \mathfrak{m}, M)=0(i<n)$, $\operatorname{Ect}_{R}^{n}(R / \mathfrak{m}, M) \neq 0([3](15 . \mathrm{B}))$. In particular, this is independent of the choice of a maximal $M$-regular sequence.

LEMma 1. Let $(R, \mathfrak{m})$ be a reduced noetherian local ring and $\mathfrak{p}_{1}, \cdots, \mathfrak{p}_{n}$

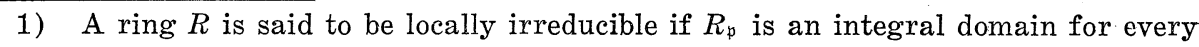
$p \in \operatorname{Spec} R$.

2) The author's original proof of this lemma was simplified by Prof. J. Nishimura as is presented here. 
$(n \geq 2)$ be the set of minimal prime ideals of $R$. Suppose that $\mathfrak{a}+\mathfrak{b}$ is $\mathfrak{m}$-primary with $\mathfrak{a}=\bigcap_{i=1}^{r} \mathfrak{p}_{i}$ and $\mathfrak{b}=\bigcap_{j=r+1}^{n} \mathfrak{p}_{j}$ for some $r(1 \leq r<n)$, then it holds that depth $R \leq 1$.

Proof. We may assume that depth $R \geq 1$. We have an exact sequence

$$
0 \longrightarrow R \longrightarrow R / \mathfrak{a} \oplus R / \mathfrak{b} \longrightarrow R /(\mathfrak{a}+\mathfrak{b}) \longrightarrow 0
$$

of $R$-modules. From this we have an exact sequence

$$
\operatorname{Hom}_{R}(R / \mathfrak{m}, R / \mathfrak{a} \oplus R / \mathfrak{b}) \longrightarrow \operatorname{Hom}_{R}(R / \mathfrak{m}, R /(\mathfrak{a}+\mathfrak{b})) \longrightarrow \operatorname{Ext}_{R}^{1}(R / \mathfrak{m}, R)
$$

of $R$-modules. Since depth $R \geq 1$, we have $\operatorname{depth}(R / \mathfrak{a}+R / \mathfrak{b}) \geq 1$ and $\operatorname{Hom}(R / \mathfrak{m}$, $R / \mathfrak{a} \oplus R / \mathfrak{b})=0$. On the other hand, $\operatorname{Hom}_{R}(R / \mathfrak{m}, R /(\mathfrak{a}+\mathfrak{b})) \neq 0$ because $\mathfrak{a}+\mathfrak{b}$ is m-primary. Therefore $\operatorname{Ext}_{R}^{1}(R / \mathfrak{m}, R) \neq 0$ and depth $R=1$.

q.e.d.

Let $R$ be a noetherian ring. By a formal fiber we mean a fiber $k(\mathfrak{p})$ $\otimes_{R_{\mathrm{m}}}\left(\widehat{R_{\mathrm{m}}}\right)$ of $R_{\mathrm{m}} \rightarrow\left(\widehat{R_{\mathrm{m}}}\right)$ with some maximal ideal $\mathfrak{m}$ of $R$ and $\mathfrak{p} \in \operatorname{Spec} R_{\mathrm{m}}$. If $\mathfrak{p}$ is a minimal prime of $R_{\mathrm{m}}$, then we call $k(\mathfrak{p}) \otimes_{R_{\mathrm{m}}}\left(\widehat{R_{\mathrm{m}}}\right)$ a genetic formal fiber.

We say that a local domain $R$ is analytically irreducible if $\hat{R}$ is an integral domain.

TheORem 1. Let $(R, \mathrm{~m})$ be a pseudo-geometric normal local domain. Then $R$ is analytically irreducible if and only if the generic formal fiber is locally irreducible.

Proof. Only if part is obvious. Let $\mathfrak{P}_{1}, \cdots, \mathfrak{P}_{n}$ be the minimal primes in $\hat{R}$. Suppose that $\hat{R}$ is not an integral domain, then $n \geq 2$ because $\hat{R}$ is reduced.

Consider a minimal prime ideal $\mathfrak{Q}$ of $\mathfrak{P}_{1}+\bigcap_{j=2}^{n} \mathfrak{P}_{j}$. We may assume that $\mathfrak{P}_{j} \subset \mathfrak{Q}$ if and only if $1 \leq j \leq r(2 \leq r \leq n)$. Then $\mathfrak{P}_{1} \hat{R}_{\mathfrak{Q}}+\bigcap_{j=2}^{r} \mathfrak{P}_{j} \hat{R}_{\mathfrak{Q}}$ is primary to $\mathfrak{\Omega} \hat{R}_{\mathfrak{a}}$ and $\mathfrak{P}_{1} \hat{R}_{\mathfrak{a}}, \cdots, \mathfrak{P}_{r} \hat{R}_{\mathfrak{Q}}$ form the set of minimal primes of $\hat{R}_{\mathfrak{a}}$. Set $\mathfrak{q}=R \cap \cong$.

1) Suppose that ht $\mathfrak{q} \geq 2$. Then depth $R_{\mathfrak{q}} \geq 2$ because $R$ is normal. Since $R_{\mathrm{q}} \rightarrow \hat{R}_{\mathfrak{Q}}$ is faithfully flat, we have depth $\hat{R}_{\mathfrak{Q}} \geq 2$, which is a contradiction by Lemma 1.

2) Suppose that ht $q=1$. Since $R$ is normal, $R_{q}$ is a discrete valuation ring with a uniformizing parameter, say $q$. If $\operatorname{dim} \hat{R}_{\mathfrak{a}}=1$, then $\hat{R}_{\mathfrak{a}} / q \hat{R}_{\mathfrak{\Omega}}$ is not reduced since $\hat{R}_{\mathfrak{a}}$ is not a regular local ring. This is a contradiction because formal fibers of psudo-geometric ring must be reduced. Thus $\operatorname{dim} \hat{R}_{0}>1$. Then $\operatorname{dim} \hat{R}_{\mathfrak{Q}} / q \hat{R}_{\mathfrak{Q}} \geq 1$. Furthermore depth $\hat{R}_{\mathfrak{Q}} / q \hat{R}_{\mathfrak{Q}}=0$ by Lemma 1 and it follows that $\hat{R}_{\mathfrak{a}} / q \hat{R}_{\mathfrak{a}}$ is not reduced; a contradiction.

3) Suppose that $\mathfrak{q}=0$. Then $\hat{R}_{\mathfrak{Q}}$ is a localization of the generic formal fiber. Since $r \geq 2, \hat{R}_{\mathfrak{a}}$ is not a domain.

Therefore if the generic formal fiber is locally irreducible, then $\hat{R}$ must 
be an integral domain.

q.e.d.

A local domain is said to be unibranched if the derived normal ring is local. In particular henselian local domain is unibranched.

Corollary. Let $R$ be a pseudo-geometric unibranched local domain. Then $R$ is analytically irreducible if and only if the generic formal fiber is locally irreducible.

Proof. Let $R^{\prime}$ be the derived normal ring of $R$. Then $R^{\prime}$ is a finite $R$-module and $\hat{R}^{\prime}=R^{\prime} \otimes_{R} \hat{R}$. Therefore generic formal fibers of $R$ and $R^{\prime}$ are the same. On the other hand, since $R \rightarrow \hat{R}$ is flat, $\hat{R} \rightarrow \hat{R}^{\prime}$ is injective. Since $R^{\prime}$ is local, we may assume that $R=R^{\prime}$, then the assertion follows from Theorem 1 .

q.e.d.

We say that a ring $R$ is equidimensional if $\operatorname{dim} R_{\mathrm{m}} / \mathfrak{p} R_{\mathrm{m}}=\operatorname{dim} R$ for all minimal prime ideals $\mathfrak{p}$ of $R$ and for all maximal ideals $\mathfrak{m}$ of $R$ such that $\mathfrak{p} \subset \mathfrak{m}$.

Theorem 2. Let $R$ be pseudo-geometric normal local domain. Then $\hat{R}$ is equidimensional if and only if the generic formal fiber is locally equidimensional.

Proof. Only if part is obvious because every complete local ring is catenary (see $\S 2$ for the definition) by [4] (34.6). Let $\mathfrak{R}_{1}, \cdots, \mathfrak{P}_{n}$ be the minimal prime ideals in $\hat{R}$. We may assume $\operatorname{dim} \hat{R} / \mathfrak{\beta}_{i}=\operatorname{dim} \hat{R}$ if and only if $1 \leq i$ $\leq r$ for some $r(1 \leq r \leq n)$. Suppose that $r<n$. Put $\mathfrak{Y}=\bigcap_{i=1}^{r} \mathfrak{P}_{i}$ and consider the set $\left\{\mathfrak{Q}_{i, 1}, \cdots, \mathfrak{Q}_{i, n_{i}}\right\}$ of minimal prime ideals of $\mathfrak{P}_{i}+\mathfrak{A}(r+1 \leq i \leq n)$. Let $\mathfrak{O}$ be a minimal member in the set $\left\{\bigcap_{i, j_{i}} ; r+1 \leq i \leq n, 1 \leq j_{i} \leq n_{i}\right\}$ by inclusion relation. We may assume that $\mathfrak{\Omega}=\mathfrak{Q}_{i, 1}$ if and only if $r+1 \leq i \leq s$ for some $s$ with $r+1 \leq s \leq n$. Then $\mathfrak{P}_{i} \hat{R}_{\mathfrak{a}}+\mathfrak{U} \hat{R}_{\mathfrak{2}}$ are primary to $\mathfrak{Q} \hat{R}_{\mathfrak{a}}$ for $r+1 \leq i \leq s$ and $\left(\cap_{i=r+1}^{s} \mathfrak{P}_{i} \hat{R}_{\mathfrak{Q}}\right)+\mathfrak{U} \hat{R}_{\mathfrak{Q}}$ is also $\cong \hat{R}_{\mathfrak{Q}}$-primary. We may assume that $\mathfrak{P}_{j}(1 \leq j \leq r)$ is contained in $\cong$ if and only if $1 \leq j \leq t$ for some $t(1 \leq t \leq r)$. Then the minimal prime ideals of $\hat{R}_{\mathfrak{Q}}$ are $\mathfrak{P}_{1} \hat{R}_{\mathfrak{Q}}, \cdots, \mathfrak{\Re}_{t} \hat{R}_{\mathfrak{Q}}, \mathfrak{P}_{r+1} \hat{R}_{\mathfrak{Q}}, \cdots, \mathfrak{P}_{s} \hat{R}_{\mathfrak{Q}}$ and $\left(\cap_{i=1}^{t} \mathfrak{P}_{i} \hat{R}_{\mathfrak{Q}}\right)+$ $\left(\cap_{j=r+1}^{s} \mathfrak{R}_{j} \hat{R}_{\mathfrak{Q}}\right)$ is $\mathfrak{Q} \hat{R}_{\mathfrak{Q}}$-primary. Therefore, by the proof of Theorem 1 , we see that $\hat{R}_{\mathfrak{Q}}$ is a localization of the generic formal fiber. Note that $\hat{R}_{\mathfrak{Q}}$ is not equidimensional because $r<n$ and $\hat{R}$ is catenary. Thus, if we assume that the generic formal fiber is locally equidimensional then we have $r=n$, that is, $R$ is equidimensional.

q.e.d.

Corollary. Let $R$ be a pseudo-geometric unibranched domain. Then $R$ is equidimensional if and only if generic formal fiber is locally equidimensional.

Proof. By the proof of Corollary to Theorem 1, it is sufficient to show 
that $\hat{R}$ is equidimensional if and only if $\hat{R}^{\prime}$ is equidimensional, and this follows from the fact that $\hat{R}$ and $\hat{R}^{\prime}$ are local and catenary.

q.e.d.

Now we define a ring extension such that it does not change geometric properties of formal fibers. Let $K$ be a field. A property $(P)$ is called geometric if it holds that a $K$-algebra $R$ enjoys $(P)$ if and only if $L \otimes_{K} R$ enjoys $(P)$ for any finite field extension $L$ of $K$. We say that a homomorphism $A \rightarrow$ $B$ of rings is an integral extension if $B$ is integral (that is, $B$ is an inductive limit of finite $A$-algebras) and dominate (that is, minimal primes of $B$ are mapped to those of $A$ and minimal primes of $A$ are in the image of Spec $B \rightarrow$ Spec $A$ ).

We say that $A \rightarrow B$ is an (f.q.)-integral extension if the following three conditions are satisfied.

1). $B$ is a ring of fractions of some integral extension of $A$.

2). Spec $B \otimes_{A}\left(\widehat{A_{\mathfrak{m}}}\right) \rightarrow \operatorname{Spec}\left(\widehat{A_{\mathrm{m}}}\right)$ is surjective for every maximal ideal $\mathfrak{n t}$ of A.

3). $A$ and $B$ are locally noetherian and for any maximal ideal $\mathfrak{R}$ of $B$, there exists a finite $A$-subalgebra $A^{\prime}$ of $B$ such that $\mathfrak{m}^{\prime}=\mathfrak{N} \cap A^{\prime}$ is maximal and $\left(\widehat{B_{\mathfrak{n}}}\right)=\left(\widehat{A_{\mathfrak{m}^{\prime}}^{\prime}}\right)$.

REMARK 1. The henselization of a local ring or a finite integral extension is an (f.q.)-integral extension.

Lemma 2. Let $R \rightarrow T$ be an (f.q.)-integral extension. Then (generic) formal fibers of $R$ are locally equidimensional, if and only if so are those of $T$.

Proof. In order to consider formal fibers, by 2) even for the proof of if part, we may assume that $R$ and $T$ are local domains with maximal ideals $\mathfrak{n}$ and $\mathfrak{n}$ respectively. Let $R^{\prime}$ be a finite $R$-subalgebra of $T$ such that $\hat{T}=\left(\widehat{R_{\mathrm{m}^{\prime}}^{\prime}}\right)$ with $\mathfrak{n t}^{\prime}=\mathfrak{n} \cap R^{\prime}$. Let $L, K^{\prime}$, and $K$ be the fields of quotients of $T, R^{\prime}$ and $R$ respectively. Then we have

$$
K^{\prime} \otimes_{R^{\prime}}\left(\widehat{R_{\mathfrak{m}^{\prime}}^{\prime}}\right)=K^{\prime} \otimes_{R^{\prime}} \hat{T}=K^{\prime} \otimes_{R^{\prime}}\left(T \otimes_{T} \hat{T}\right)=L \otimes_{T} \hat{T}
$$

So we may assume moreover that $T$ is a localization of a finite extension $R^{\prime}$ of $R$. Then the generic formal fiber of $T$ coincides with a direct summand of

$$
L \otimes_{R^{\prime}}\left(R^{\prime} \otimes_{R} \hat{R}\right)=L \otimes_{R} \hat{R}=L \otimes\left(K \otimes_{R} \hat{R}\right) .
$$

Now the assertion follows from the fact that equidimensionality of $K$ algebra $K \otimes_{R} \hat{R}$ is geometric.

REMARK 2. By the above investigation, the lemma holds even if equi- 
dimensionality is replaced by other geometric properties (for example, geometrically regular, geometrically reduced or Cohen Macaulay, etc.).

\section{§II. Integrally solvable obstructions}

Let $\mathfrak{p} \subset \mathfrak{q}$ be prime ideals of a ring $R$. We say that a chain $\mathfrak{p}=\mathfrak{p}_{0} \subset \mathfrak{p}_{1} \subset \ldots$ $\subset \mathfrak{p}_{r}=\mathfrak{q}$ of prime ideals $\mathfrak{p}_{j}(0 \leq j \leq r)$ is maximal, if there is no prime ideal $\mathfrak{p}^{\prime}$ such that $\mathfrak{p}_{i-1} \subset \mathfrak{p}^{\prime} \subset \mathfrak{p}_{i}(1 \leq i \leq r)$. We say

$1^{\circ}$. $R$ is catenary if for any primes $\mathfrak{p} \subset q$, ht $\mathfrak{q} / \mathfrak{p}$ is finite and is equal to the length of any maximal chain between $\mathfrak{p}$ and $q$.

$2^{\circ}$. $R$ is universally catenary if any finitely generated $R$-algebra is catenary.

$3^{\circ}$. $R$ satisfies the second chain condition if every integral extension of $R$ is equidimensional and catenary.

$4^{\circ}$. A local ring $R$ is formally equidimensional if $\hat{R}$ is equidimensional.

The following is called the chain conjecture. [7]

The Chain Conjecture. Let $R$ be noetherian domain, then any pair of prime idials $\mathfrak{p} \subset \mathfrak{q}$ in the derived normal ring $R^{\prime}$ of $R, R_{\mathfrak{q}}^{\prime} / \mathfrak{p} R_{\mathfrak{q}}^{\prime}$ satisfies the second chain condition.

The known basic result is Proposition 1 below, here we say that $B$ is an $A$-algebra of essentially finite type, if $B$ is a ring of fractions of a finitely generated $A$-algebra.

Proposition 1. (Nagata)

(1) If a noetherian local ring $R$ is formally equidimensional, then $R$ satisfies the second chain condition.

(2) Let a local domain $S$ be of essentially finite type over a noetherian ring $R$. If $R$ satisfies the second chain condition, then so does $S$. In particular $R$ is universally catenary.

Proof. For the proof, see [4] (34.6) and [6] for (1) and (2), or see [10] (Prop. 1.5).

In order to obtain our main result, Theorem 3 below, we show some propositions. The following lemma is known and is an easy consequence of the going up theorem ([4] (10.9)).

LEMma 3. Let $R \rightarrow S$ be an integral extension of rings. If $S$ is equidimensional, then so is $R$. If moreover $S$ is catenary, then so is $R$.

Proposition 2. ${ }^{3)} \quad$ Let $R$ be a noetherian local domain. Then $R$ is univer-

3) Proposition 2 and Proposition 3 follow from [10] (1.9) and (1.11). But we can give simple proofs as follow in our situation. 
sally catenary if and only if $R$ satisfies the second chain condition.

Proof. The if part follows from Proposition 1. Suppose that $R$ is universally catenary. Then the derived normal ring $R^{\prime}$ of $R$ is equidimensional because dimension formula holds between $R$ and $T$ by [3] (14.c), where $T$ is a finite $R$-subalgebra of $R^{\prime}$ such that for any maximal ideal $\mathfrak{m}$ of $T$, there exists a unique maximal ideal $\mathfrak{n}$ of $R^{\prime}$ lying over $\mathfrak{m}$. The existence of such a $T$ follows from [4] (33.10). Therefore any integral extension of a universally catenary local domain is equidimensional by the going down theorem ([4] (10.13)) and Lemma 3.

Suppose that an integral extension $S$ of $R$ is not catenary. Let $\mathfrak{Q} \subset \mathfrak{P}$ be primes of $S$ such that ht $\mathfrak{P} / \mathfrak{Q}$ is minimum in the pairs of primes such that

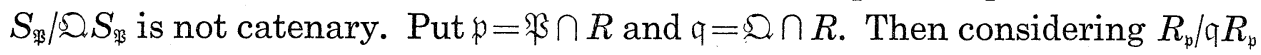

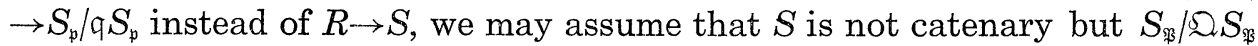
is catenary for any pair $\mathfrak{\subset} \subset \mathfrak{B}$ of prime ideals in $S$ such that $\mathfrak{Q}$ is not minimal or $\mathfrak{P}$ is not maximal. Then there exists a prime $\mathfrak{P}$ of $S$ such that ht $\mathfrak{P} / \mathfrak{Q}_{+}$ ht $\mathfrak{N} / \mathfrak{\beta}<\operatorname{dim} S$ for some minimal prime $\mathfrak{Q}$ and some maximal ideal $\mathfrak{N}$ of $S$.

Since $R$ is catenary, it holds that ht $\mathfrak{p}>$ ht $\mathfrak{P} / \mathfrak{Q}$ or $\operatorname{dim} R / \mathfrak{p}>$ ht $\mathfrak{N} / \mathfrak{R}$ with $\mathfrak{p}=\mathfrak{P} \cap R$; this contradicts the equidimensionality of $S_{\mathfrak{p}}$ and $S / \mathfrak{p} S$ because $R_{\mathfrak{p}}$ $\rightarrow S_{\mathfrak{p}}$ and $R / \mathfrak{p} \rightarrow S / \mathfrak{p} S$ are integral extensions.

q.e.d.

Proposition 3. Let $(R, \mathfrak{m})$ be a pseudo-geometric henselian local domain. If $R$ is not formally equidimensional, then $R$ is not catenary.

Proof. Let $\Omega_{1}, \cdots, \mathfrak{\Omega}_{s}$ be the minimal prime ideals of $\hat{R}$ such that $r=$

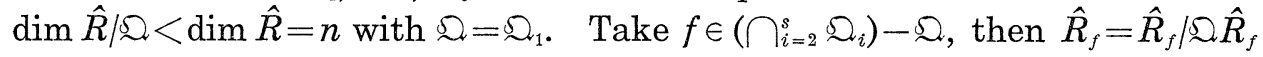
is a domain of dimension $r-1$. Let $x_{1}, \cdots, x_{r-1} \in \mathfrak{m}$ be a set of elements such that $f, x_{1}, \cdots, x_{r-1}$ form a system of parameters of $\hat{R} / \Omega$. Then $x_{1}, \cdots, x_{r-1}$ form a system of parameters of some maximal ideal $\mathfrak{B}^{\prime}$ of $\hat{R}_{f}$.

Set $\mathfrak{P}=\hat{R} \cap \mathfrak{P}^{\prime}$ and $\mathfrak{p}=R \cap \mathfrak{P}$. Then $\mathfrak{Q}$ is the unique minimal prime of $\hat{R}$ contained in $\mathfrak{P}$, and therefore $\mathfrak{P}$ is a minimal prime of $\left(x_{1}, \cdots, x_{r-1}\right) \hat{R}$ and of $\mathfrak{p} \hat{R}$. Therefore ht $\mathfrak{p}=\mathrm{ht} \mathfrak{\Re}=r-1$ by [4] (22.9). Note that $\operatorname{dim} \hat{R} / \mathfrak{R}=1$. We shall show that $\operatorname{dim} R / \mathfrak{p}=1$ which implies Proposition 3 because ht $\mathfrak{m} / \mathfrak{p}=1$, ht $\mathfrak{p}=r-1$ and $\operatorname{dim} R=n>r$.

Suppose $\operatorname{dim} R / \mathfrak{p} \geq 2$. Set $S=R / p$ and let $S^{\prime}$ be the derived normal ring of $S$. Since $R$ and therefore $S$ is henselian, $S^{\prime}$ is a normal local ring of $\operatorname{dim} S^{\prime}$ $\geq 2$ and therefore depth $S^{\prime} \geq 2$. But $\hat{S}^{\prime}=S^{\prime} \otimes_{S} \hat{R} / \mathfrak{p} \hat{R}$ because $S^{\prime}$ is a finite $S$-module. The fact that $\mathfrak{P}$ is a minimal prime of $\mathfrak{p} \hat{R}$ and that $\operatorname{dim} \hat{R} / \mathfrak{B}=1$ contradicts depth $\hat{S}^{\prime} \geq 2$. q.e.d.

Let $R$ be a ring. An $R$-algebra $R^{\prime}$ is called the normalization of $R$ at $\mathfrak{p}(\in \operatorname{Spec} R)$ if $R^{\prime}$ is the integral closure of $R / \mathfrak{p}$ in $k(\mathfrak{p})$. If $\mathfrak{p}$ is a minimal prime 
of $R$, then we call $R^{\prime}$ a generic normalization. A localization of a (generic) normalization by its maximal ideal is called a (generic) local normalization.

By a henselization of $R$, we mean the henselization with respect to some maximal ideal of $R$.

Under the circumstances, we can state our main result as follows.

THEOREM 3. Let $R$ be a pseudo-geometric ring. Then the following are equivalent to each other.

1). Every generic formal fiber of $R$ is locally equidimensional.

2) Every generic local normalization of $R$ is formally equidimensional.

3). Every generic normalization of $R$ is universally catenary.

4). Every henselization of $R$ is catenary.

Proof. 1) $\Rightarrow 2$ ) follows from Theorem 2 and Lemma 2.

2) $\Rightarrow 3$ ) follows from Proposition 1.

$3) \Rightarrow 4)$ : Let $H$ be a henselization of $R, \mathfrak{P}$ a minimal prime of $H$ and $\mathfrak{p}=$ $\mathfrak{R} \cap R$. Let $H^{\prime}$ and $R^{\prime}$ be the derived normal rings of $H / \Re$ and $R / \mathfrak{p}$ respectively, then we have a natural injection $R^{\prime} \rightarrow H^{\prime}$. Then $H^{\prime}$ is catenary by Proposition 2 because $H^{\prime}$ is a localization of some integral extension of the universaly catenary ring $R^{\prime}$. Therefore $H / \Re$ is catenary by Lemma 3, because $H^{\prime}$ is a local domain. Since $\mathfrak{B}$ is an arbitrary minimal prime of $H, H$ is catenary.

4) $\Rightarrow 1$ ): Let $\mathfrak{m}$ be a maximal ideal of $R$ and $H$ the henselization of $R_{\mathrm{m}}$. Then generic formal fibers of $H$ are locally equidimensional by Proposition 3 and Corollary to Theorem 2. Therefore generic formal fibers of $R_{\mathrm{m}}$ are locally equidimensional by Lemma 2 . Since $\mathfrak{m}$ is arbitrary, we see that (1) holds.

q.e.d.

Corollary 1 . If $R$ enjoys the conditions in Theorem 3, then any $R$-algebra of essentially finite type also satisfies those conditions.

Proof. An easy consequence of Proposition 1.

Corollary 2. Let $R$ be a pseudo-geometric domain. Then the Chain Conjecture holds for $R$ if (and only if) $R$ satisfies the conditions in Theorem 3.

REMARK 3. The existence of a ring with integrally solvable obstruction is shown by Nagata in [4] (Example 2). Indeed it is easy to see that the ring is a $G$-ring (cf. [3] (33.A)) if the field $K$ is of characteristic zero.

\section{$\S$ III. Construction of a ring with a formal obstruction}

The following example is a pseudo-geometric local domain of dimension 3 whose generic formal fiber is not locally equidimensional. Therefore the 
chain conjecture does not hold by Theorem 3 .

Let $\boldsymbol{Q}$ be the rational number field, $\left\{a_{i}, b_{j}, c_{k} ; i, j, k \in N\right\}$ be a set of indeterminates where $N$ is the set of natural numbers. Set

$$
K=Q\left(\left\{a_{i}, b_{j}, c_{k} ; i, j, k \in N\right\}\right)
$$

and let $x, y, z, w$ be indeterminates.

Let $\mathscr{P}(\subset K[x, y, z, w])$ be a set of prime elements in $S=K[x, y, z, w]_{(x, y, z, w)}$ such that $x, y, z, w \in \mathscr{P}$ and such that each principal prime ideal of $S$ has a unique generator in $\mathscr{P}$. Since $\mathscr{P}$ is countable, elements of $\mathscr{P}$ can be numbered in such a way that $\mathscr{P}=\left\{p_{i} ; i \in N\right\}$ with $p_{1}=w$.

We set

$$
\begin{array}{ll}
q_{n}=\prod_{k=1}^{n} p_{k}, \quad g_{n}=x+\sum_{k=1}^{n} a_{k} q_{k}^{k}, & h_{n}=y+\sum_{k=1}^{n} b_{k} q_{k}^{k}, \quad l_{n}=z+\sum_{k=1}^{n} c_{k} q_{k}^{k} \\
\mathfrak{p}_{n}=\left(g_{n}, h_{n}, 1_{n}\right) S \quad(n \in N) & \text { and } \mathfrak{p}_{0}=(x, y, z) S .
\end{array}
$$

Then $\mathfrak{p}_{n}$ is a prime ideal of height 3 because $g_{n}, h_{n}, l_{n}$ form a part of regular system of parameters. We want to prove

THEOREM 4. With the notation above, there is an enumeration of $\mathscr{P}$ such that $p_{n} \notin \mathfrak{p}_{n-1}$ for all $n \in N$.

Before proving Theorem 4, we need some preliminaries.

Lemma 4. With $S$ and $\mathscr{P}$ as above, take elements $\alpha\left(\in \mathfrak{m}-\mathfrak{m}^{2}\right), \beta(\in \mathfrak{m}=$ $(x, y, z, w) S), p_{1}, \cdots, p_{n}(\in \mathscr{P})$ and $n_{0}(\in N)$. Let $\mathfrak{q}_{0}, \mathfrak{q}_{1}, \cdots, \mathfrak{q}_{r}$ be prime ideals of $S$ such that ht $\mathfrak{q}_{i} \leq 3(0 \leq i \leq r), \alpha \in \mathfrak{q}_{0}$ and $(\alpha, \beta) S \not \mathfrak{q}_{j}$ for any $j(1 \leq j \leq r)$. Then there exists $p^{\prime} \in \mathscr{P}$ such that $p^{\prime} \neq p_{i}(1 \leq i \leq n), p^{\prime} \oplus \mathfrak{q}_{0}$ and $\alpha+\beta p^{\prime n_{0}} \oplus \mathfrak{q}_{j}(1 \leq j \leq r)$.

Proof. We may assume that all $\mathfrak{q}_{i}(0 \leq i \leq r)$ are primes of height 3 . We may also assume that $\alpha \in \mathfrak{q}_{j}$ if and only if $0 \leq j \leq s$ for some $s(0 \leq s \leq r)$. Let $v_{j}(s+1 \leq j \leq r)$ be the valuation defined by a discrete valuation ring which is a localization of the derived normal ring of $S / \mathfrak{q}_{j}$. Let $\alpha_{j}(s+1 \leq j \leq r)$ be the class of $\alpha$ in $S / \mathfrak{q}_{j}$ and set $m=\max \left\{2, v_{s+1}\left(\alpha_{s+1}\right), \cdots, v_{r}\left(\alpha_{r}\right)\right\}$. Take $\gamma \in \mathfrak{m}-$ $\bigcup_{i=0}^{r} q_{i}$, then $\gamma$ is not an associate of $\alpha$. So it is easy to see that the set $\{\alpha+$ $\left.\gamma^{l} ; l \geq m\right\}$ is a set of mutually non-associate prime elements. Therefore there exists $l_{0} \geq m$ such that $\alpha+\gamma^{l_{0}}$ is not an associate of any $p_{k}(1 \leq k \leq n)$.

Let $p^{\prime}$ be the element of $\mathscr{P}$ such that $p^{\prime}$ is an associate of $\alpha+\gamma^{l_{0}}$. We shall show that $p^{\prime} \notin \mathfrak{q}_{0}$ and $\alpha+\beta p^{\prime n_{0}} \notin \mathfrak{q}_{j}(1 \leq j \leq r)$.

That $p^{\prime} \notin \mathfrak{q}_{0}$ is obvious because $p^{\prime}=\varepsilon\left(\alpha+\gamma^{l_{0}}\right), \alpha \in \mathfrak{q}_{0}, \gamma \notin \mathfrak{q}_{0}$ and $\varepsilon$ is a unit of $S$.

As for $\alpha+\beta p^{\prime n_{0}}$ we have two cases;

1) If $1 \leq j \leq s$, then $p^{\prime} \notin \mathfrak{q}_{j}$ as above, and $\alpha+\beta p^{n_{0}} \notin \mathfrak{q}_{j}$ because $\alpha \in \mathfrak{q}_{j}, \beta \notin \mathfrak{q}_{j}$. 
2). Assume that $s+1 \leq j \leq r$. The class $\delta_{j}$ of $\beta p^{\prime n_{0}}$ modulo $\mathfrak{q}_{j}$ is not congruent to $-\alpha_{j}$ because

$$
v_{j}\left(\delta_{j}\right)=v_{j}\left(\beta_{j}\right)+n_{0} v_{j}\left(\alpha_{j}+\gamma_{j}^{l_{0}}\right)>v_{j}\left(\alpha_{j}\right)
$$

where $\beta_{j}$ and $\gamma_{j}$ are the classes of $\beta$ and $\gamma$ modulo $\mathfrak{q}_{j}$ respectively. Therefore we have $\alpha+\beta p^{\prime n_{0}} \notin \mathfrak{q}_{j}$.

Now for each $n \in N$, we denote by $[1, n]$ the set $\{1,2, \cdots, n\}$. We say that $\psi_{n}:[1, n] \rightarrow \mathscr{P}$ is a good map if $\psi_{n}$ is injective and $p_{k} \oplus \mathfrak{p}_{k-1}(1 \leq k \leq n)$ with $p_{k}=\psi_{n}(k)$.

Now suppose that we have already defined a good map $\psi_{n}$ and that we are given an element $p \in \mathscr{P}$ such that $p \neq p_{k}=\psi_{n}(k)(1 \leq k \leq n)$. We want to extend $\psi_{n}$ to a good map $\psi_{m}(m>n)$ such that $\psi_{m}(m)=p$.

To do this we set

$$
\begin{aligned}
& G_{n}(U, V)=g_{n}+a_{n+1}\left(q_{n} U\right)^{n+1}+a_{n+2}\left(q_{n} U V\right)^{n+2} \\
& H_{n}(U, V)=h_{n}+b_{n+1}\left(q_{n} U\right)^{n+1}+b_{n+2}\left(q_{n} U V\right)^{n+2} \quad \in S[U, V] \\
& L_{n}(U, V)=l_{n}+c_{n+1}\left(q_{n} U\right)^{n+1}+c_{n+2}\left(q_{n} U V\right)^{n+2}
\end{aligned}
$$

with indeterminates $U, V$. Then we have linear combinations of these elements having neither $U$ nor $U V$ as follows.

$$
\begin{aligned}
& F_{n}(U)=\left(b_{n+2} g_{n}-a_{n+2} h_{n}\right)+\left(b_{n+2} a_{n+1}-a_{n+2} b_{n+1}\right)\left(q_{n} U\right)^{n+1} \\
& I_{n}=\left(b_{n+1} c_{n+2}-b_{n+2} c_{n+1}\right) g_{n}+\left(a_{n+2} c_{n+1}-a_{n+1} c_{n+2}\right) h_{n}+\left(a_{n+1} b_{n+2}-a_{n+2} b_{n+1}\right) l_{n}
\end{aligned}
$$

Since any 1-minor or 2-minor of the matrix $\left(\begin{array}{lll}a_{n+1} & b_{n+1} & c_{n+1} \\ a_{n+2} & b_{n+2} & c_{n+2}\end{array}\right)$ is a unit of $S[U, V]$, we have

$$
\left(G_{n}, H_{n}, L_{n}\right) S[U, V]=\left(G_{n}, F_{n}, I_{n}\right) S[U, V] .
$$

Now we have

Proposition 4. Suppose that a good map $\psi_{n}:[1, n] \rightarrow \mathscr{P}$ and an element $p \in \mathscr{P}\left(p \neq p_{k}=\psi_{n}(k)\right.$ for any $k$ up to $\left.n\right)$ are given. Then there exists a good extension $\psi_{m}:[1, m] \rightarrow \mathscr{P}$ of $\psi_{n}$ with $n \leq m \leq n+3$ such that $p \notin \mathfrak{p}_{m}$.

Proof. 1) Suppose that $p$ is an associate of $I_{n}$. Then there exists $p^{\prime} \in$ $\mathscr{P}$ such that $p^{\prime} \neq p_{k}(1 \leq k \leq n)$ and $p^{\prime} \notin \mathfrak{p}_{n}$ by Lemma 4 with $\alpha=g_{n}, \mathfrak{q}_{0}=\mathfrak{p}_{n}$ and $r=0$. We set $p_{n+1}=p^{\prime}$. (This means that we define $\psi_{n+1}$ with $\psi_{n+1}(n+1)=p^{\prime}$ and $\psi_{n+1}(k)=\psi_{n}(k)$ for $\left.1 \leq k \leq n\right)$. Then $\psi_{n+1}$ is a good map and $p$ is not an associate of $I_{n+1}$ because $I_{n}$ and $I_{n+1}$ form a part of regular system of parameters of $S$. Therefore, replacing $n$ by $n+1$ if necessary, we may assume $p$ is not an associate of $I_{n}$.

2) If $p \notin \mathfrak{p}_{n}$ then $\psi_{n}$ itself is the required map. 
3) Assume that $p \in \mathfrak{p}_{n}$. Consider a minimal prime $q$ of $\left(p, I_{n}\right)$ in $S$. Then ht $\mathfrak{q}=2$ and $\mathfrak{q} \nsupseteq\left(\alpha, q_{n}\right) S$ with $\alpha=b_{n+2} g_{n}-a_{n+2} h_{n}$.

Indeed, otherwise we have $\mathfrak{q} \ni p_{k}$ for some $k(1 \leq k \leq n)$ and therefore $\mathfrak{q}+$ $g_{n} S \supseteq\left(g_{n}, h_{n}, l_{n}, p_{k}\right) S$, that is, $\mathfrak{q}+g_{n} S \supseteq \mathfrak{p}_{k-1}+p_{k} S$. Since $\mathfrak{p}_{k-1}$ is a prime ideal of height 3 and $p_{k} \notin \mathfrak{p}_{k-1}$ by assumption we have ht $\left(\mathfrak{q}+g_{n} S\right) \geq 4$, which contradicts the fact that ht $\mathfrak{q}=2$.

Therefore there exists $p^{\prime} \in \mathscr{P}$ such that $p^{\prime} \notin \mathfrak{p}_{n}, p^{\prime} \neq p_{k}(1 \leq k \leq n)$ and $p, I_{n}$, $F_{n}\left(p^{\prime}\right)$ form a regular sequence of $S$ by Lemma 4 with $\mathfrak{q}_{0}=\mathfrak{p}_{n}, \mathfrak{q}_{i}(1 \leq i \leq r)$ the minimal prime ideals of $\left(p, I_{n}\right) S, \beta=\left(b_{n+2} a_{n+1}-a_{n+2} b_{n+1}\right) q_{n}^{n+1}$ and $n_{0}=n+1$. We define $p_{n+1}$ to be $p^{\prime}$.

4) If $p \notin \mathfrak{p}_{n+1}$ then $\psi_{m}=\psi_{n+1}$ is the required map.

5) Assume that $p \in \mathfrak{p}_{n+1}$. Let $\mathfrak{q}$ be a minimal prime of $\left(p, I_{n}, F_{n}\left(p_{n+1}\right)\right) S$. If $\mathfrak{q} \ni g_{n+1}=g_{n}+a_{n+1}\left(q_{n} p_{n+1}\right)^{n+1}=G_{n}\left(p_{n+1}, 0\right)$, then

$\mathfrak{q} \supseteq\left(I_{n}, F_{n}\left(p_{n+1}\right), G_{n}\left(p_{n+1}, 0\right)\right) S=\mathfrak{p}_{n+1}$ and since ht $\mathfrak{q}=3=$ ht $\mathfrak{p}_{n+1}$, we have $\mathfrak{q}$ $=\mathfrak{p}_{n+1}$ and $\mathfrak{q} \nexists q_{n} p_{n+1}=q_{n+1}$. Therefore there exists $p^{\prime \prime} \in \mathscr{P}$ such that $p^{\prime \prime} \notin \mathfrak{p}_{n+1}$, $p^{\prime \prime} \neq p_{k}(1 \leq k \leq n+1)$ and $p, I_{n}, F_{n}\left(p_{n+1}\right), G_{n}\left(p_{n+1}, p^{\prime \prime}\right)$ form a regular sequence of $S$ by Lemma 4 with $\mathfrak{q}_{0}=\mathfrak{p}_{n+1}, \mathfrak{q}_{j}(1 \leq i \leq r)$ the minimal primes of $\left(p, I, F_{n}\left(p_{n+1}\right)\right) S$, $n_{0}=n+2, \alpha=g_{n+1}=g_{n}+a_{n+1}\left(q_{n} p_{n+1}\right)^{n+1}$ and $\beta=a_{n+2} q_{n+1}^{n+2}$.

We define $p_{n+2}$ to be $p^{\prime \prime}$. Then since $p_{n+2}=\left(I_{n}, F_{n}\left(p_{n+1}\right), G_{n}\left(p_{n+1}, p_{n+2}\right)\right) S$, $p$ is a nonzerodivisor for $S / \mathfrak{p}_{n+2}$ and we have $p \notin \mathfrak{p}_{n+2}$.

Therefore $\psi_{m}=\psi_{n+2}$ is the required extension.

q.e.d.

Proof of the Theorem 4. Using Proposition 3, we obtain a good enumeration for $\mathscr{P}$ in Theorem 4 by induction.

Construction of an example.

Let $\varphi: N \rightarrow \mathscr{P}$ be an enumeration of $\mathscr{P}$ in Theorem 4. Set $p_{n}=\psi(n)$, then $p_{n} \notin$ $\mathfrak{p}_{n-1}(n \in N)$.

Let $g, h, l$ be the elements of the completion $\hat{S}=K[[x, y, z, w]]$ of $S$ defined by

$$
g=x+\sum_{n=1}^{\infty} a_{n} q_{n}^{n}, \quad h=y+\sum_{n=1}^{\infty} b_{n} q_{n}^{n}, \quad l=z+\sum_{n=1}^{\infty} c_{n} q_{n}^{n}
$$

We set also

$$
\sigma_{n}=q_{n}^{-n} \sum_{k=n}^{\infty} a_{k} q_{k}^{k}, \quad \tau_{n}=q_{n}^{-n} \sum_{k=n}^{\infty} b_{k} q_{k}^{k} \text { and } \lambda_{n}=q_{n}^{-n} \sum_{k=n}^{\infty} c_{k} q_{k}^{k} \quad(n \in N)
$$

which are elements of $\hat{S}$, because

$$
q_{n}^{-n} q_{k}^{k}=q_{n}^{k-n}\left(p_{n+1} \cdots p_{k}\right)^{k} \in S \quad \text { if } k \geq n .
$$

Let $L$ be the field of fractions of $S$ and we define 


$$
\omega_{n}=g_{n} h_{n} / q_{n}^{n}, \quad \mu_{n}=g_{n} l_{n} / q_{n}^{n} \in L \quad(n \in N) .
$$

Then we have relations

$$
\begin{aligned}
& \omega_{n}=q_{n} p_{n+1}^{n+1}\left(\omega_{n+1}-a_{n+1} h_{n+1}-b_{n+1} g_{n+1}+a_{n+1} b_{n+1} q_{n+1}^{n+1}\right) \\
& \mu_{n}=q_{n} p_{n+1}^{n+1}\left(\mu_{n+1}-a_{n+1} l_{n+1}-c_{n+1} g_{n+1}+a_{n+1} c_{n+1} q_{n+1}^{n+1}\right) .
\end{aligned}
$$

We set

$$
R^{\prime}=S\left[\left\{\omega_{n}, \mu_{m} ; n, m \in N\right\}\right], \quad R=R_{(x, y, z, w)}^{\prime} \subset L .
$$

Then we claim;

(1). $R$ is noetherian.

Indeed let $\mathfrak{p}$ be a non-zero prime of $R$, then we have $\mathfrak{p} \cap S \neq 0$ because $R$ and $S$ have the same field of fractions. Let $p(\in \mathfrak{p} \cap S)$ be a prime element of $S$. Then since $p$ is an associate of $p_{n}=\psi(n)$ for some $n \in N, R / p R$ is a homomorphic image of $S$ and hence noetherian. Therefore $\mathfrak{p}$ is finitely generated and $R$ is noetherian. ([4] (3.4))

Next we claim:

(2). $(g, h, l) \hat{S} \cap S=(0)$.

Proof. Assume for a moment that $\mathfrak{p}=(g, h, l) \hat{S} \cap S$ is not zero, then we choose a prime element $p=p_{n} \in \mathscr{P}$ such that $p_{n} \in \mathfrak{p}$. Then

$$
\left(g_{n-1}, h_{n-1}, l_{n-1}\right) S \subseteq \mathfrak{p} \quad \text { and } \quad p_{n} \notin\left(g_{n-1}, h_{n-1}, l_{n-1}\right) S=\mathfrak{p}_{n-1}
$$

by our construction. Since $\mathfrak{p}_{n-1}$ is a prime ideal of height 3 , we have ht $\mathfrak{p} \geq$ 4 , which contradicts ht $(g, h, l) \hat{S}=3$ in view of [4] (22.9).

REMARK 4. (2) means, in particular, that $g, h, l$ are algebraically independent over $L$. For, otherwise, one sees easily that $\mathfrak{p}$ above is not zero in view of the fact that $g, h, l$ form a part of regular system of parameters of $\hat{S}$.

(3). $R$ is S-isomorphic to $K[[x, y, z, w]] /(g h, g l)$.

Proof. Consider the diagram, where $i$ and $j$ are canonical injections and $p$ is the canonical projection. Since we have relations

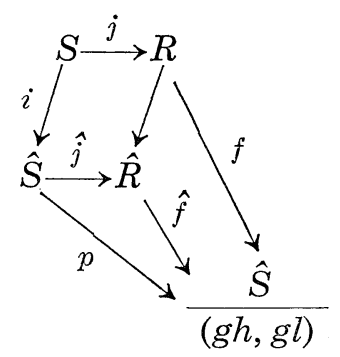




$$
\begin{aligned}
& g_{n} h_{n}=g h-q_{n+1}^{n+1}\left(g \tau_{n+1}+h \sigma_{n+1}-q_{n+1}^{n+1} \sigma_{n+1} \tau_{n+1}\right) \\
& g_{n} l_{n}=g l-q_{n+1}^{n+1}\left(g \lambda_{n+1}+l \sigma_{n+1}-q_{n+1}^{n+1} \sigma_{n+1} \lambda_{n+1}\right)
\end{aligned}
$$

in $\hat{S}$ and since $T=\hat{S} /(g h, g l)$ is a torsion free $S$-module by (2), we have a homomorphism $f: R \rightarrow T$ such that $p \circ i=f \circ j$ defined by

$$
\begin{array}{ll}
f\left(\omega_{n}\right)=\text { class of }-q_{n} p_{n+1}^{n+1}\left(g \tau_{n+1}+h \sigma_{n+1}-q_{n+1}^{n+1} \sigma_{n+1} \tau_{n+1}\right) & \text { in } T \\
f\left(\mu_{n}\right)=\text { class of }-q_{n} p_{n+1}^{n+1}\left(g \lambda_{n+1}+l \sigma_{n+1}-q_{n+1}^{n+1} \sigma_{n+1} \lambda_{n+1}\right) & \text { in } T .
\end{array}
$$

Since $T$ is complete and $f$ is local, $f$ induces a map $\hat{f}: \hat{R} \rightarrow T$. On the other hand $\hat{j}: \hat{S} \rightarrow \hat{R}$ is surjective, because $S / \mathfrak{m}^{n} \rightarrow R / \mathfrak{m}^{n} R$ is surjective for all $n \in N$.

By the relation $\left(^{*}\right)$ we have $\hat{j}(g h) \in q_{n}^{n} \hat{R}$. Since $n$ is arbitrary, we have $\hat{j}(g h)=0$. Similarly we have $\hat{j}(g l)=0$. Therefore we have maps

$$
\hat{S} /(g h, g l) \stackrel{\hat{j}^{\prime}}{\longrightarrow} \hat{R} \stackrel{\hat{f}^{\prime}}{\longrightarrow} \hat{S} /(g h, g l)
$$

where $\hat{j}^{\prime}$ is a surjection and $f \circ \hat{j}^{\prime}$ is identity. Therefore $\hat{f}$ is an $S$-isomorphism.

(4). $R$ is pseudo-geometric.

Proof. The generic formal fiber is geometrically reduced by (3) and by $\operatorname{ch} R=0$. Since for any non-zero prime $\mathfrak{p}$ of $R, R / \mathfrak{p}$ is isomorphic to a homomorphic image of $S$, a formal fiber at $\mathfrak{p}$ is geometrically regular. So $R$ is pseudo-geometric ([2] (7.6.4)).

Consequently we have

Theorem 5. There exists a pseudo-geometric local domain whose generic formal fiber is not locally equi-dimensional. In particular The Chain Conjecture does not hold in general.

Proof. $\quad \hat{R}_{(g, h, l)}$ is a localization of a generic formal fiber of $R$ by (2) and is not equi-dimensional by (3).

q.e.d.

\section{Appendix}

In Theorem 1 and 2 (consequently Theorem 3 ) in $\S 1$, the assumption that $R$ is pseudo-geometric is essential. We shall show in this appendix that there exists a (non pseudo-geometric) noetherian normal local domain such that

1). The generic formal fiber is locally irreducible, hence locally equidimensional. Moreover every formal fiber is locally equidimensional.

2). The ring is not formally equidimensional. In particular, it is not analytically irreducible.

3). The ring is not catenary. 
The example is similar to that of $\S 3$. Before constructing it, we need some preliminaries.

Lemma 5. Let $R$ be a regular local ring of $\operatorname{dim} R \geq 3$ and $a, x, y$ be elements of $R$ such that $x$ and $y$ form a part of regular system of parameters and $a \notin(x, y) R$. Let $U, V$ be indeterminates over $R$, then

$$
\mathfrak{p}=\left(a U-x^{2}, a V-x y, x V-y U\right) R[U, V]
$$

is a prime ideal of $R[U, V]$.

Proof. It is easy to see that for any minimal prime $q$ of $\mathfrak{p}$, we have ht $q$ $=2$. We have a primary decomposition

$$
p+V R[U, V]=\left(a U-x^{2}, x y, y U, V\right)=\left(a U-x^{2}, y, V\right) \cap(x, U, V)
$$

where $\left(a U-x^{2}, y, V\right)$ is a prime ideal of height 3 in $R[U, V]$ by assumption. Therefore $V$ is not in any minimal prime of $p$ and hence $p$ is a radical ideal.

Since minimal primes of $\mathfrak{p}+a R[U, V]$ are those of $(a, x, y) R[U, V]$ and those of $(a, x, U) R[U, V]$, which are of height $3, a$ is a non zero-divisor for $\mathfrak{p}$. Then it is easy to see that $\mathfrak{p} R_{a}[U, V]=\left(a U-x^{2}, a V-x y\right) R_{a}[U, V]$ is a prime ideal. Therefore $\mathfrak{p}$ is a prime ideal of $R[U, V]$.

q.e.d.

Proposition 5. Let $R$ be regular local ring of $\operatorname{dim} R \geq 4$ and $a, x, y, z$ be elements of $R$ such that $x, y, z$ form a part of regular system of parameters of $R$ and $a \oplus(z, x, y) R$. Let $U$ and $V$ be indeterminates and let

$$
q=(a U-x(x+z), a V-x y,(x+z) V-y U) A
$$

be an ideal of $A=R[U, V]$. Then $\mathfrak{q}+z A$ is a prime ideal of $A$ and $A / \mathfrak{q}$ is a normal domain.

Proof. $q+z A$ is a prime ideal of $A$ by Lemma 5 , which is of height 3 . In particular $\mathfrak{q}$ is a prime of $A$ because $h t \mathfrak{q}=2$. Consider the ideal $\mathfrak{a}=(a, x$, $y, z) A$ which is of height 4 by our assumption. Let $\mathfrak{p}$ be a prime ideal of $A$ such that $\mathfrak{p} \supseteq \mathfrak{q}$. If $\mathfrak{p}$ contains $\mathfrak{a}$, then $\operatorname{depth} A_{p} / \mathfrak{q} A_{p} \geq 2$ because $\mathfrak{q}+z A$ is a prime. If $\mathfrak{p}$ does not contain $\mathfrak{a}$, then it is easy to see that $q A_{\mathfrak{p}}$ is generated by a part of regular system of parameters of $A_{\mathfrak{p}}$ of length two and $A_{\mathfrak{p}} / \mathfrak{q} A_{\mathfrak{p}}$ is a regular local ring. Therefore, $A / \mathfrak{q}$ is a normal domain by [3] (Th. 39).

q.e.d.

Let $S$ and $\mathscr{P}$ be the same as those of $\S 3$. Put $\mathscr{P}^{\prime}=\mathscr{P}-\{z\}$. Then we have

THEOREM $4^{\prime}$. With the notation above, there exists a numbering $\psi^{\prime}: N \rightarrow \mathscr{P}^{\prime}$ such that $p_{n}^{\prime} \notin \mathfrak{q}_{n-1}(n \in N)$ where $p_{1}^{\prime}=\psi^{\prime}(1)=w, p_{n}^{\prime}=\psi^{\prime}(n), q_{n}^{\prime}=\prod_{k=1}^{n} p_{k}^{\prime}, g_{n}^{\prime}=x+$ $\sum_{k=1}^{n} a_{k} q_{k}^{\prime k}, h_{n}^{\prime}=y+\sum_{k=1}^{n} b_{k} q_{k}^{\prime k}, q_{0}=(x, y, z) S$ and $q_{n}=\left(g_{n}^{\prime}, h_{n}^{\prime}, z\right) S$. 
Proof. An easy consequence of the proof of Theorem 4.

Let $g^{\prime}$ and $h^{\prime}$ be the elements of $\hat{S}=K[[x, y, z, w]]$ defined by

$$
g^{\prime}=x+\sum_{n=1}^{\infty} a_{n} q_{n}^{\prime n}, \quad h^{\prime}=y+\sum_{n=1}^{\infty} b_{n} q_{n}^{\prime n} .
$$

Let $L$ be the quotient field of $S$ and we set

$$
\alpha_{n}=g_{n}^{\prime}\left(z+g_{n}^{\prime}\right) / q_{n}^{\prime n}, \quad \beta_{n}=g_{n}^{\prime} h_{n}^{\prime} / q_{n}^{\prime n} \in L \quad(n \in N) .
$$

Then we have relations

$$
\begin{aligned}
& \alpha_{n}=q_{n}^{\prime} p_{n+1}^{\prime n+1}\left(\alpha_{n+1}-a_{n+1} z-2 a_{n+1} g_{n+1}^{\prime}+a_{n+1}^{2} q_{n+1}^{\prime n+1}\right) \\
& \beta_{n}=q_{n}^{\prime} p_{n+1}^{\prime n+1}\left(\beta_{n+1}-a_{n+1} h_{n+1}^{\prime}-b_{n+1} g_{n+1}^{\prime}+a_{n+1} b_{n+1} q_{n+1}^{\prime n+1}\right) .
\end{aligned}
$$

We set

$$
T_{n}=S\left[\alpha_{n}, \beta_{n}\right]_{\left(x, y, z, w, \alpha_{n}, \beta_{n}\right)}, \quad T=\underline{\lim } T_{n} \subset L .
$$

Then there is an $S$-homomorphism

$$
f_{n}: A_{n}=S[U, V] /\left\{q_{n}^{\prime n} U-g_{n}^{\prime}\left(z+g_{n}^{\prime}\right), q_{n}^{\prime n} V-g_{n}^{\prime} h_{n}^{\prime},\left(z+g_{n}^{\prime}\right) V-h_{n}^{\prime} U\right\} \longrightarrow S\left[\alpha_{n}, \beta_{n}\right]
$$

defined by $f_{n}(u)=\alpha_{n}, f_{n}(v)=\beta_{n}$ where $u$ and $v$ are classes of $U$ and $V$ in $A_{n}$ respectively. By construction we have

$$
q_{n}^{\prime n} \notin\left(g_{n}^{\prime}, h_{n}^{\prime}, z\right) S=q_{n} .
$$

Therefore $A_{n}$ is a normal domain and $z$ is a prime element of $A_{n}$ by Proposition 5. In particular, $f_{n}$ is an isomorphism. of $T$.

Therefore $T$ is a local normal domain such that $z$ is a prime element

Now we claim:

(1). $T$ is noetherian.

Proof. Let $\mathfrak{p}$ be a non-zero prime ideal of $T$.

a). If $\mathfrak{p} \cap S=z S$, then it is easy to see that $T_{\mathfrak{p}}=S_{q}$ with $q=z S$. Since $z T$ is prime, we see $\mathfrak{p}=z T$.

b). If $\mathfrak{p} \cap S \neq z S$, then there exists a prime element $p \in \mathfrak{p} \cap S$ of $S$ such that $p \in \mathscr{P}^{\prime}$. Then $T / p T$ is a homomorphic image of $S$, hence noetherian. So $\mathfrak{p}$ is finitely generated.

By a) and b), $T$ is noetherian ([4] (3.4)).

(2). $\quad\left(g^{\prime}, h^{\prime}, z\right) \hat{S} \cap S=z S, \quad\left(z+g^{\prime}, h^{\prime}\right) \hat{S} \cap S=0, \quad g^{\prime} \hat{S} \cap S=0$.

Proof. Easy by construction. 
(3). $\hat{T} \simeq \hat{S} /\left\{g^{\prime}\left(z+g^{\prime}\right), g^{\prime} h^{\prime}\right\}$.

Proof. $\hat{S} /\left\{g^{\prime}\left(z+g^{\prime}\right), g^{\prime} h^{\prime}\right\}$ is a torsion free $S$-module by (2). Therefore the assertion follows from the proof of (3) in $\S 3$.

(4). The generic formal fiber of $T$ is locally irreducible and formal fibers of $T$ are locally equidimensional.

Pro.of. $\mathfrak{p}_{1}=g^{\prime} \hat{T}$ and $\mathfrak{p}_{2}=\left(z+g^{\prime}, h^{\prime}\right) \hat{T}$ are minimal primes of $\hat{T}$ such that $\mathfrak{p}_{1} \cap \mathfrak{p}_{2}=0$ by (3). Since $\mathfrak{p}_{1}\left(L \otimes_{T} \hat{T}\right)+\mathfrak{p}_{2}\left(L \otimes_{T} \hat{T}\right)=L \otimes_{T} \hat{T}$, we have $L \otimes_{T} \hat{T}$ is locally irreducible.

Formal fiber at $z T$ is locally equidimensional because $\hat{T} / z \hat{T}$ has only one minimal prime. Formal fibers at other non-zero primes are also locally equidimensional because they are regular.

(5). Tis not catenary.

Proof. Let $\mathfrak{p}=(x+z, y, w) T$, then ht $\mathfrak{p}=1$ by [4] (22.9) because $\mathfrak{p} \hat{T}$ is a prime ideal of height 1 in $\hat{T}$. On the other hand $\operatorname{dim} T / \mathfrak{p}=1 . \quad$ ht $\mathfrak{p}+\operatorname{dim} T / \mathfrak{p}$ $=2<\operatorname{dim} T=3$. Therefore $T$ is not catenary.

REMARK 5. We can also construct following examples;

(a). Pseudo-geometric regular local ring whose generic formal fiber is not geometrically normal.

(b). Analytically irreducible noetherian local domain $R$ such that the

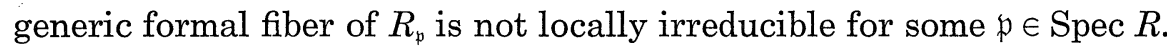

\section{References}

[1] D. Ferrand and M. Raynaud, Fibers formelles d'un anneau local noetherien, Ann. Sci. École Norm. Sup., 3 (1970), 295-311.

[2] A. Grothendieck, E.G.A. IV, I.H.E.S. Publ. Math., 24 (1965).

[ 3 ] H. Matsumura, Commutative algebra, Benjamin, Inc., New York (1970).

[4] M. Nagata, Local rings, John Wiley, New York (1962). Reprinted. Krieger, Huntington, N.Y. (1975).

[5] M. Nagata, On the chain problem of prime ideals, Nagoya Math. J., 10 (1956), 51-64.

[6] M. Nagata, Note on a chain condition of prime ideals, Mem. Coll. Sc. Univ. Kyoto, Series A Math., 32 (1959), 85-90.

[ 7 ] L. J. Ratriff, Jr., Chain conjectures in ring theory, Lect. Notes in Math. no. 647, Springer-Verlag, Berlin Heidelberg New York, 1978.

[ 8 ] L. J. Ratriff, Jr., On quasi-unmixed local domains, the altitude formula, and the chain condition for prime ideals (I), Amer. J. Math., 91 (1969), 508-528. 
[9] C. Rotthaus, Universell Japanische Ringe mit nicht offenem regulärem Ort (to appear).

[10] H. Seydi, Anneaux henseliens et conditions de chaines, Bull. Soc. Math. France, 98 (1970), 9-31.

\section{Department of MATHEMatics \\ KYOTO UNIVERSITY \\ КYOTO 606 \\ JAPAN}

